\title{
Metastatic Colorectal Cancer: Lessons Learned, Future Possibilities
}

\author{
Presented by Alan P. Venook, MD
}

\section{Abstract}

Survival of patients with metastatic colorectal cancer has dramatically improved over the past 20 years, primarily because physicians have become adept at using the many regimens approved for this patient population. Future advances may come from understanding molecular subtypes, finding and treating new actionable mutations, and harnessing the immune system.

J Natl Compr Cancer Netw 2016;14(5.5):666-668

At the NCCN 21st Annual Conference, Alan P. Venook, MD, from UCSF Helen Diller Family Comprehensive Cancer Center, looked back for "lessons learned" in the treatment of metastatic colorectal cancer (mCRC) and peered into the future to gauge how the field is evolving. mCRC research and new treatment strategies can be informed by what has already been learned, said Dr. Venook, the Madden Family Distinguished Professor of Medical Oncology and Translational Research.

Advances in outcomes can be measured by the dramatic change in overall survival (OS). A meta-analysis from 1992 - a time when treatment was almost exclusively 5 -fluorouracil—showed median OS to be only 8 months. ${ }^{1}$ Approximately 20 years later, OS exceeds 30 months (Figure 1). ${ }^{2}$

Many of the advances in outcomes have come from the identification of patient subsets that will benefit most from a specific treatment. For example, the discovery of the RAS mutation and its importance in excluding treatment in patients who harbor RAS for agents that target the epidermal growth factor receptor is the

Presented by Alan P. Venook, MD, Department of Medicine, UCSF Helen Diller Family Comprehensive Cancer Center, San Francisco, California.

Dr. Venook has disclosed that he receives grant/research support from Bayer HealthCare; Bristol-Myers Squibb Company; Eli Lilly and Company; Genentech, Inc.; Merck \& Co., Inc.; and Roche Laboratories, Inc.

Correspondence: Alan P. Venook, MD, UCSF Helen Diller Family Comprehensive Cancer Center, 1450 3rd Street, HD 376, Box 3111, San Francisco, CA 94143-3111. E-mail: Alan.Venook@ucsf.edu best example of treatment refinement that improves survival—but only for the selected subset. Imaging with CT scans to find and treat metastatic disease earlier has also made a difference in survival, he said.

The introduction of new agents, however, has not increased outcomes for progression-free survival, for which median control-of-disease time hovers around 10 months.

"Colon cancer is a complex disease, and we have made progress by doing a lot of things well," Dr. Venook suggested. The biggest benefit has come from physicians being "more nimble" in using "the tools on hand," he said. "What is important is to strategize: what will I do next, and how can I spare patients toxicity?" Further, Dr. Venook emphasized that "It matters more that we do a variety of things, rather than being hyper-aggressive."

\section{What Has Worked, and What Has Not}

Lessons have also been learned from what has not worked. One important hypothesis proved wrong was that a drug that works well in mCRC would also be effective in the adjuvant setting. In the NSABP and AVANT studies, ${ }^{3,4}$ bevacizumab did not improve outcomes in patients with stage II or III disease, nor did cetuximab in the N0147 trial for patients with stage III KRAS wild-type disease. ${ }^{5}$

"The paradigm we have historically used across all diseases - that if a drug is active in metastatic disease we will see activity in the adjuvant setting, and cure patients who would not otherwise experience a cure-did not turn out to be the case," Dr. Venook stated. 


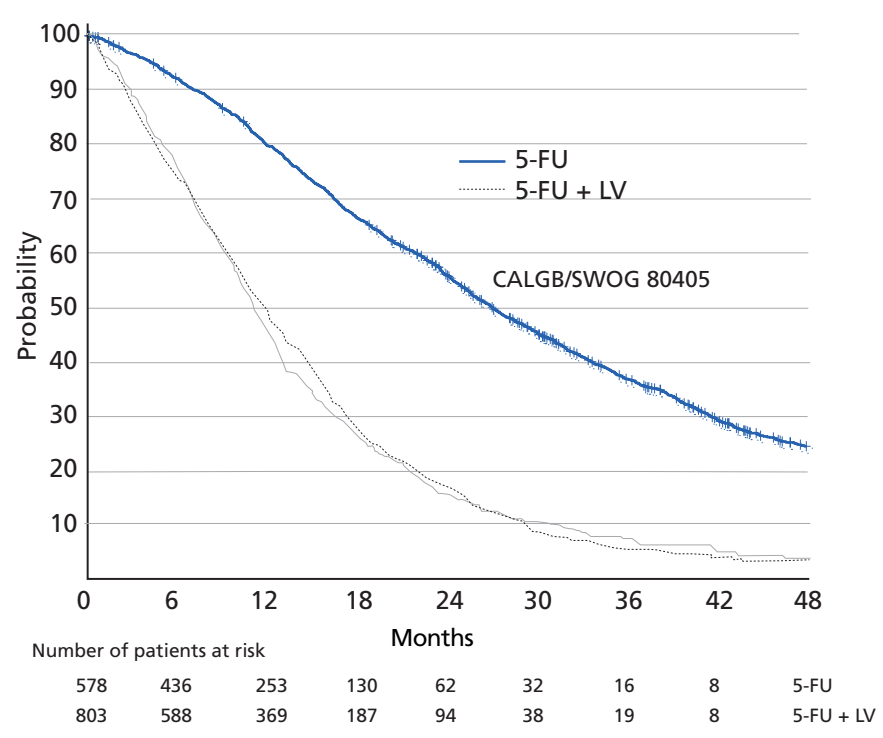

Figure 1. Colorectal cancer: 20 years later. Abbreviations: 5-FU, 5-fluorouracill; LV, leucovorin.

Another lesson learned has been regarding inconsistent findings. In the European FIRE-3 trial, OS was longer with combination cetuximab and chemotherapy compared with bevacizumab and chemotherapy. ${ }^{6}$ However, this finding was not substantiated in CALGB/SWOG 80405, in which median OS was approximately 32 months for both cetuximab and bevacizumab in combination with chemotherapy in all patients with RAS wild-type disease. ${ }^{?}$

Therefore, in the continuum of care, the optimal strategy is decided not from the start but "down the road," during which time the physician observes the disease and the patient and adjusts treatment accordingly (Figure 2). "With multiple lines of treatment and maintenance, we are getting more treatment to the patient and that's probably making a difference," he said.

\section{Novel Strategies Being Tried}

There are novel strategies that appear effective and are gaining traction, but their effect on OS remains unknown. One of these novel strategies is selective internal radiation therapy (SIRT), which is yttrium-90-labeled resin microspheres that deliver a single large radiation dose to liver tumors. In a study reported at the 2015 ASCO Annual Meeting, SIRT plus FOLFOX and bevacizumab yielded a 7.9-month improvement in progression-free survival. ${ }^{8}$
"Radiation to the liver controls liver metastases effectively, but patients still succumb to extra-hepatic disease," noted Dr. Venook, who said that pooled results from ongoing studies will be informative.

\section{Take Stock and Figure Out Next Steps}

The future holds "big strides" in terms of understanding tumor biology, improved staging, appreciation of lifestyle impact, advances in ablative and surgical techniques, and development of effective new drugs (used earlier in disease), including immune modulators, Dr. Venook predicted.

It is now known that exercise, aspirin, and a non-Western diet are associated with a decreased risk of disease recurrence; aspirin is associated with a reduced risk of death; and sweetened beverages are associated with an increased risk of recurrence. Additionally, a low baseline vitamin D level is a poor prognostic sign for patients with metastatic cancer. Such knowledge "empowers patients to be in control of their outcomes," he pointed out. However, Dr. Venook noted that it is not known if repletion of vitamin D is a simple way to improve outcomes, based on the results of CALGB/SWOG 80405 in which patients with the highest baseline levels had a 35\% improvement in OS $(P=.001){ }^{9}$

Also on the horizon is a greater appreciation of intratumoral heterogeneity as revealed by multiregion gene sequencing and an increasing knowledge of the key signaling pathways, their interaction, and the drugs that target them. These insights will help drive drug development and treatment selection.

"Because of complex interactions, it's unlikely that any one molecule will make a difference," he acknowledged. Additionally, gene expression-based molecular profiles will stratify patients into 4 consensus molecular subtypes that may eventually become a new staging system. "Clearly, there are molecular features defining this disease, and the different biologic features related to genes may tell us which patients will do well and which won't," he said.

A better understanding of molecular subtypes and signaling pathways is already informing drug development and treatment selection. Dr. Venook predicted the next actionable mutation in $\mathrm{mCRC}$ will be HER2, which is overexpressed in approximately $5 \%$ of patients. Combinations of anti-HER2 agents are currently being evaluated in this patient subgroup. 


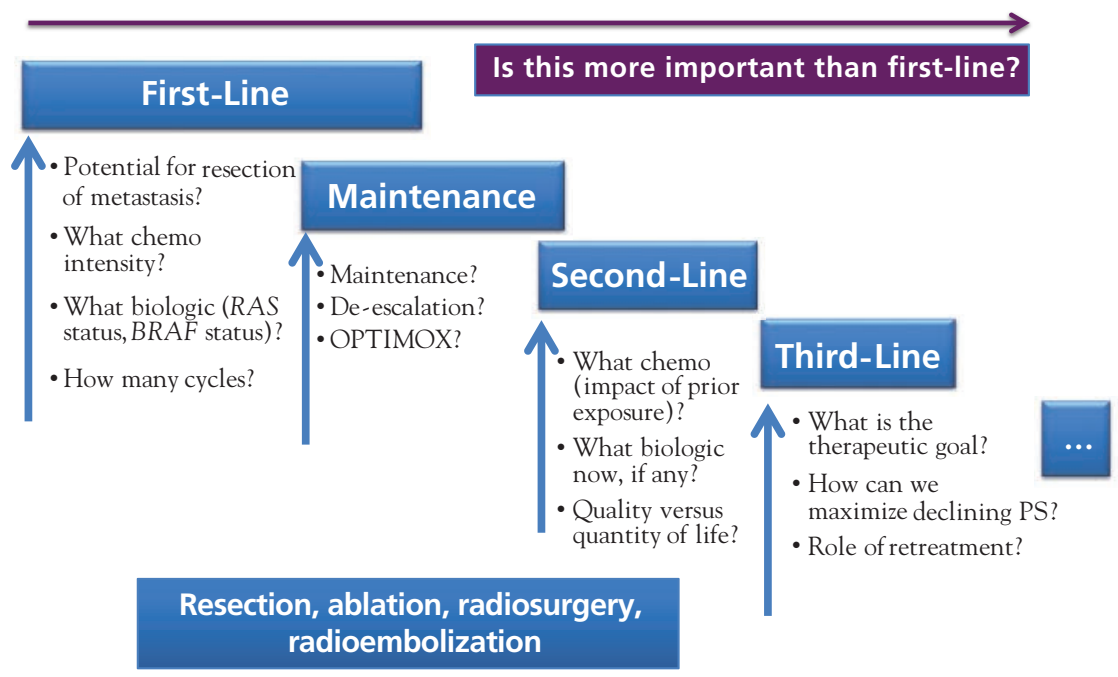

Figure 2. Continuum of care in metastatic colorectal cancer: decisions, decisions, decisions.

Abbreviations: chemo, chemotherapy; PS, performance status.

A very encouraging step forward has been the discovery that the immune system can also be harnessed for therapeutic benefit in the subset of patients with $\mathrm{mCRC}$ and microsatellite instability (MSI)high tumors. "Dramatic advances related to immune checkpoint inhibitors have changed the game," he said. In the study by Le et $a,{ }^{10}$ the anti-PD-1 agent pembrolizumab produced immune-related responses in $62 \%$ of patients.

"We have seen patients rise from the ashes, so we recommend MSI testing for all patients with advanced CRC at the outset," he said. "I suspect checkpoint inhibitors will be approved soon for this indication - they are remarkable drugs in this 3\% of patients with mCRC."

He also called for greater efforts to be directed toward screening for CRC. In a study in Delaware, an enhanced screening program substantially improved screening rates, reduced the diagnosis of CRC at advanced stages, and reduced mortality by $41 \%{ }^{11}$

Finally, Dr. Venook emphasized the need to appreciate tumor biology according to tumor location within the colon. Right-sided tumors carry a worse prognosis, possibly related to their different embryologic origin, microbiome, or molecular features. Location seems to be a biomarker for outcome that has virtually been ignored, he said, adding that data from an analysis of CALGB/SWOG 80405 will be presented at the 2016 ASCO Annual Meeting.

\section{References}

1. Modulation of fluorouracil by leucovorin in patients with advanced colorectal cancer: evidence in terms of response rate. Advanced Colorecta Cancer Meta-Analysis Project. J Clin Oncol 1992;10:896-903.

2. Venook AP, Niedzwiecki D, Lenz HJ, et al. CALGB/SWOG 80405: phase III trial of irinotecan/5-FU/leucovorin or oxaliplatin/5-FU/leucovorin with bevacizumab or cetuximab for patients with KRAS wild-type untreated metastatic adenocarcinoma of the colon or rectum [abstract]. J Clin Oncol 2014;32(Suppl):5s. Abstract LBA3.

3. Allegra CJ, Yothers G, O'Connell MJ, et al. Phase III trial assessing bevacizumab in stages II and III carcinoma of the colon: results of NSABP protocol C-08. J Clin Oncol 2011;29:11-16.

4. De Gramont A, Van Cutsem E, Schmoll HJ, et al. Bevacizumab plus oxaliplatin-based chemotherapy as adjuvant treatment for colon cancer (AVANT): a phase 3 randomised controlled trial. Lancet Oncol 2012;13:1225-1233.

5. Alberts SR, Sargent DJ, Nair S, et al. Effect of oxaliplatin, fluorouracil, and leucovorin with or without cetuximab on survival among patients with resected stage III colon cancer: a randomized trial. JAMA 2012;307:13831393.

6. Heinemann V, von Weikersthal LF, Decker T, et al. FOLFIRI plus cetuximab versus FOLFIRI plus bevavcizumab as first-line treatment for patients with metastatic colorectal cancer (FIRE-3): a randomized, openlabel, phase 3 trial. Lancet Oncol 2014;15:1065-1075.

7. Lenz H, Niedzwiecki D, Innocenti F, et al. CALGB/SWOG 80405: phase III trial of irinotecan/5-FU/leucovorin (FOLFIRI) or oxaliplatin/5-FY/ leucovorin (mFOLFOX6) with bevacizumab or cetuximab for patients with expanded RAS analyses untreated metastatic adenocarcinoma of the colon or rectum [abstract]. Ann Oncol 2014;25(Suppl 4):Abstract 5010.

8. Gibbs P, Heinemann V, Sharma NK, et al. SIRFLOX: randomized phase III trial comparing first-line mFOLFOX $6+/$ - bevacizumab versus mFOLFOX $6+$ selective internal radiation therapy $+/$ - bevacizumab in patients with metastatic colorectal cancer [abstract]. J Clin Oncol 2015;33(Suppl):Abstract 3502.

9. $\mathrm{Ng} \mathrm{K}$, Venook AP, Sato K, et al. Vitamin D status and survival of metastatic colorectal cancer patients: results from CALGB/SWOG 80405 (Alliance) [abstract]. J Clin Oncol 2015;33(Suppl):Abstract 507.

10. Le DT, Uram JN, Wang H, et al. PD-1 blockade in tumors with mismatch repair deficiency [abstract]. J Clin Oncol 2015;33(Suppl):Abstract LBA100.

11. Grubbs SS, Polite BN, Carney J Jr, et al. Eliminating racial disparities in colorectal cancer in the real world: it took a village. J Clin Oncol 2013;31:1928-1930. 